\title{
Review
}

\section{Medicinal Herb Research: Serum Pharmacological Method and Plasma Pharmacological Method}

\author{
Jinwen Ge, ${ }^{a}$ Dongsheng Wang, ${ }^{b}$ Rong He ${ }^{c}$ Huibin Zhu, ${ }^{a}$ Yuhong WAng,${ }^{a}$ and Shilin $\mathrm{HE}^{*}, d$ \\ ${ }^{a}$ Department of Vascular Biology Lab, Hunan University of Chinese Medicine; Changsha 410007, China: \\ ${ }^{b}$ Department of Integrative Medicine, Xiangya Hospital/Central South University; Changsha 410008, China: \\ ${ }^{c}$ Department of Pathology, University Hospital and Clinics, University of Wisconsin; Madison, Wisconsin 53192, \\ U.S.A.: and ${ }^{d}$ Hemostasis Physiology Lab, Xiangya Medical School/Central South University; Changsha 410078, China. \\ Received January 25, 2010; accepted May 26, 2010; published online June 17, 2010
}

\begin{abstract}
Serum pharmacological method has generally been used in herb studies. However, preparation of test serum for ex vivo experiment is an intricate process: besides pretreatment (heat or chemicals), it involves the proteolytic cascades of coagulation along with fibrinolysis, complement and kinin systems, as well as platelet and leukocyte activation resulting in release reactions. These processes deviate serum sample components away from the original in vivo state, and possibly also have effects on the absorbed herbal components and their downstream effectors in blood. The conclusions drawn from serum pharmacological method are at least partially uncertain in its validity. These processes can be avoided by anticoagulation. Compared to those of the serum, constituents of plasma are better reflectors of the in vivo physiological/pathological state and medicinal herb-induced changes. Therefore, we have advocated the adoption of plasma pharmacological method in ex vivo experiments of herb studies. Recent studies including our work demonstrated that the constituents and biological activities are partially different between absorbed medicinal herbs in plasma and serum. This review summarizes the experimental evidence supporting the feasibility of plasma pharmacological method and discusses the reasons and facts that flaw the serum pharmacological method. But serum pharmacological method can be used if anticoagulants interfere with experiments. It should be emphasized that the domination between plasma and serum pharmacological methods is different depending on the usage. Indeed, the pros and cons of both methods as well as the appropriate choices of coagulants in different ex vivo experimental settings remain to be further elucidated.
\end{abstract}

Key words pharmacology; experiment method; medicinal herb; ex vivo; serum; plasma

\section{INTRODUCTION}

Pharmacological experiments can be categorized into three types: in vivo, in vitro, and ex vivo. Drug therapeutic effects and adverse/toxic reactions are mainly identified by in vivo tests/trials. Elucidation of pharmacological mechanism usually depends upon analysis of in vitro and/or ex vivo results in addition to in vivo observation. Most natural medicinal herbs contain complex components, and their pharmacokinetics remains unknown. It is difficult to investigate their pharmacological actions and mechanisms in vitro by directly adding herbal powder or crude extracts into an isolated organ or cultured cells or enzymes for multiple reasons: first, direct addition of crude herbal preparations can change important extracellular parameters (e.g. ionic strength, osmotic pressure and $\mathrm{pH}$ value etc.); second, the crude preparations contain ingredients that are not absorbed in vivo and therefore not in circulation when taken orally, which may interfere with the ex vivo test system; third, many herbal medications exert their effects through metabolites after undergoing series of biotransformation; and the important step of biotransformation is absent in the ex vivo system. It is therefore more reasonable to use the cell-free portion of a blood specimen, which contains the absorbed medicinal herb components and their metabolites, in pharmacological experiments in vitro.

However, there are two major preparations of the cell-free portion of blood specimens: plasma and serum. Plasma is the virtually cell-free supernatant of blood containing anticoagulant after centrifugation. Serum is the undiluted extracellular portion of blood after adequate coagulation is completed. Serum pharmacological method, using serum with herbs as drug source for ex vivo experiments, was proposed by Iwama and his coworkers in 1987.1) To date, several thousand articles based upon this method has been published in various

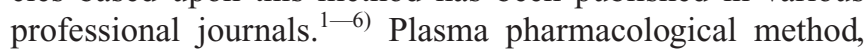
i.e. using plasma with herbs as drug source in ex vivo experiments, was proposed by us in early days of the 21 st century. ${ }^{7,8)}$ Recent work by our and other groups has demonstrated the feasibility of plasma pharmacological method in ex vivo studies of medicinal herbs, and the results obtained were partially different from those of serum pharmacological method. ${ }^{9)}$ This review should contribute to our understanding of the principal reasons and facts leading to the uncertainty of serum pharmacological method and summarize the cumulating experimental evidence supporting the feasibility of plasma pharmacological method.

\section{DIFFERENCES BETWEEN SERUM AND PLASMA}

Serum differs from plasma in many aspects since blood coagulation involves series of intricate biochemical changes and multiple cellular responses. ${ }^{10,11)}$ The major differences between serum and plasma are as follows:

Activation of Coagulation and Related Systems In plasma coagulation factors, FXII, prekallikrein, FXI, FIX, FX, prothrombin, and FXIII are proenzymes; in cofactors, high molecular weight kininogen, FVIII and FV are inactive form. During clotting, coagulation factors are converted into enzymes and cofactors are changed to active form. Blood coagulation is essentially a series of enzymatic conversions culminating in the generation of thrombin and subsequent fibrin formation, several coagulation factors are consumed (fibrino- 
gen, prothrombin, FV, FVIII, and FXIII) and many peptide fragments are generated. Once coagulation cascade is activated, anticoagulation proteins (antithrombin, heparin cofactor II, tissue factor pathway inhibitor, etc.) soon bind to coagulation serine proteases to form complexes. Contact activation of coagulation also activates fibrinolysis, kinin and complement systems. Fibrinolysis results in the generation of a series of fibrin(ogen) degradation products besides forming plasmin/antiplasmin complex. At the same time, several kinins and activated complement components (C5a, C3a) are robustly produced. ${ }^{12)}$

Activation of Blood Cells The coagulation serine proteases (especially thrombin and FXa) are strongly activate platelets and leukocytes via binding the protease-activated receptors (PARs). Platelet activation results in release of $\mathrm{Ca}^{2+}$, small bioactive molecules (ADP, ATP, 5-HT, Thromboxane $\mathrm{A}_{2}$, etc.), and proteins including growth factors (Platelet Derived Growth Factor (PDGF), Connective Tissue Activating Peptide III, etc.), coagulation factors (FV, FXI, etc.), adhesive proteins (P-selectin, vitronectin, etc.) and lysosomal enzymes (heparatinase, hydralase, etc.). Recent studies have demonstrated that platelet secretome activated by thrombin contains more than 300 different proteins. ${ }^{13)}$ Leukocyte activation causes upregulated expression of proinflammatory cytokines (Tumor Necrosis Factor (TNF)- $\alpha$, Interleukin (IL)-6, MCP-1), adhesive proteins (CD11/CD18, Lselectin), and release of lysosomal enzymes (cathepsin, elastase). In addition, thrombin causes generation and release of oxygen free radicals from neutrophils and nitric oxide from platelets. Moreover, coagulation and complement activation stimulate erythrocytes and increase their metabolism and permeability resulting in the decrease of glucose and the increase of small substances (e.g. $\mathrm{K}^{+}$, ADP, AMP, etc.) in serum.

Changes of Proteins and Nucleic Acid Thrombin, plasmin and some lysosomal enzymes can degrade certain proteins and peptides (e.g. ACTH, PTH, IL-7, IL-10, etc.). The amount of proteins/peptides in serum is significantly higher than that in plasma. Recent peptidomic analysis demonstrated 3000-7000 mass spectrometric signals in serum, corresponding to $1500-3000$ individual peptides, out of which more than $40 \%$ are serum-specific peptides and not detectable in plasma. ${ }^{14)}$ Moreover, complete coagulation injures blood cells, leading to the content increment of RNA and DNA in serum.

The coagulation-induced changes may interfere with biochemical and pharmacological experiments, but they can be avoided by proper anticoagulation.

\section{UNCERTAINTY OF SERUM PHARMACOLOGICAL METHOD}

Despite the steady increment of medicinal herb research articles based upon serum pharmacological method, it does not mean that the serum pharmacological method is infallible. Indeed, conclusions in some published papers are at least open to doubt due to the undeniable deviation of serum constituents from the in vivo circulating plasma state. The major reasons and facts leading to the uncertainty of conclusions dawned from serum pharmacological method are discussed below:
Serum Does Not Exist in Vivo Serum pharmacological actions do not reflect the real process induced by medicinal herbs in vivo. As discussed above, serum is the liquid portion of blood after complete coagulation. Under normal circumstances, in donors/patients not suffering from disseminated intravascular coagulation, the circulating cell-free portion of blood is plasma, not serum, i.e. there is no serum in vivo in the general population. One of the basic principles of ex vivo medical experiments is to keep the test samples in conditions as close to in vivo state as possible. In this aspect, serum pharmacological method is obviously flawed.

Serum Herbal Components Deviate from the in Vivo State To generate serum samples, coagulation must occur. The aftermath of coagulation is a serum-unique milieu with activated and amplified proteolytic systems which differ significantly from the in vivo plasma state. There are activated serine proteases generated from the coagulation process, lysosomal enzymes released from activated platelets and leukocytes, as well as activated complements and plasmin in the serum sample. It is without doubt that certain herb components, their metabolites, and downstream effectors are prone to enzymatic degradation. On the other hand, it is also highly likely that certain inactive components are now cleaved and converted into active substances in this serumunique milieu. In addition, some components of herb and their metabolites could adhere to blood clot and/or blood cells, and are eliminated during clot removal (Fig. 1). Multifold artifacts happen as a result of serum converting from plasma. It is highly doubtful that serum pharmacological method is the optimal method to monitor and detect what is happening to the herb components in vivo and it is reasonable to conclude that the ex vivo bioactivities of serum samples deviates from those of in vivo plasma containing the same absorbed medicinal herbs.

Serum Pretreatment Causes Changes In order to eliminate the inherent bioactivity of serum, pretreatment of serum sample is commonly carried out prior to usage of the samples in ex vivo experiments. It usually involves heat or chem-

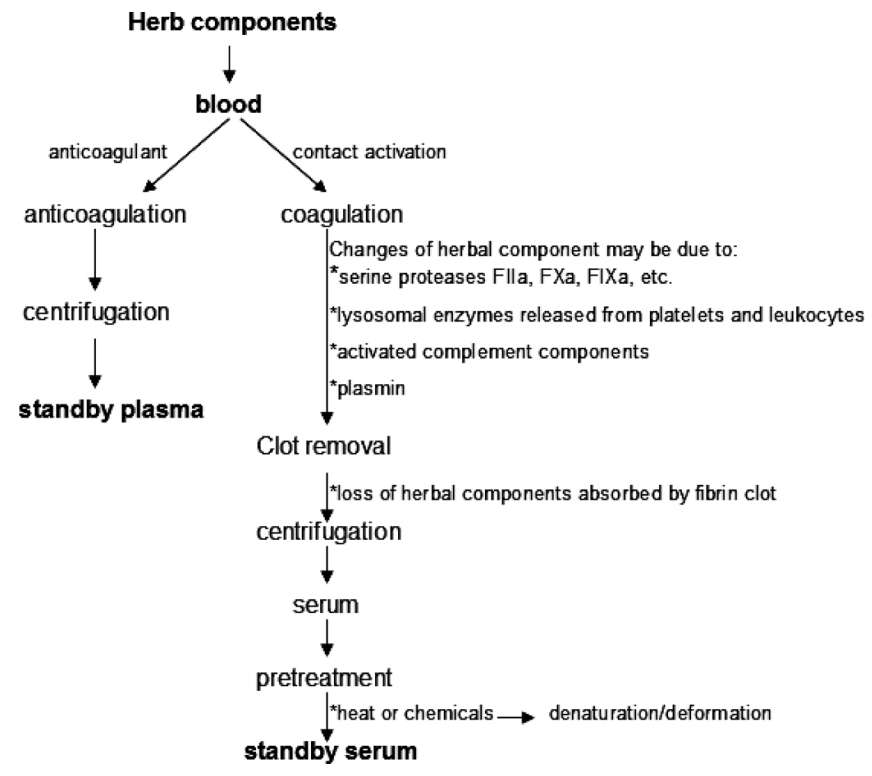

Fig. 1. Possible Influences of Serum Preparation Processes on Herba Components and Endogenous Respondent Agents in Blood 
ical precipitation treatment (e.g. acetone, ethanol). Indeed, these pretreatments can not completely denature all proteins especially glycoproteins containing higher carbohydrate percentages such as prothrombin, FVII, FIX, etc. In addition, these procedures do not discriminate between endogenous and exogenous substances. They not only inactivate complements and enzymes as targeted, but also possibly deform/ denature certain herbal components and their downstream bioactive substances as by-products (Fig. 1). Recent studies have shown significant differences in 36 proteins between plasma and heat-inactivated plasma, and 19 proteins between heat-inactivated serum and plasma. ${ }^{15}$ It is not difficult to see that pretreatment of serum adds variability and artifacts to the samples, and further flaws the serum pharmacological method.

Difficulty of Setting Up Appropriate Controls for Serum Samples Proper control group is a crucial and indispensable component in a valid experimental design. Welldesigned and appropriate control groups contain confounding factors comparable to those of the test groups, so that the effects of the test factor stand out. It is very difficult to set up the proper control for a serum sample containing absorbed medicinal herbs in reality, because many herbs could have certain enhancing or inhibitory effects on coagulation, fibrinolysis, complement, as well as platelet and leukocyte activation. This intricate background milieu in which the absorbed herb components are suspended can not be simply mimicked by a usual "pooled normal serum." Doing so could result in actual incompatibility between the control and testing groups. For example, if the purpose of a study is to examine a herb's effect on vascular endothelial cells (VEC) proliferation, and the herb tested does have a positive effect on VEC proliferation, but it also has anticoagulant effect at the same time; the stimulatory effect of the herb on VEC proliferation may be confounded by its anticoagulant effect, since the latter causes decreased platelet activation and growth factors (PDGF, VEGF) release by decreasing thrombin generation. This confounding anticoagulant effect of the herb on coagulation would be absent in the control serum group, leading to false negative conclusion.

\section{FEASIBILITY OF PLASMA PHARMACOLOGICAL METHOD}

Plasma is used in most pharmacokinetic studies to avoid the influence of blood coagulation on drugs. This principle should also be applicable to medicinal herbal studies. Promisingly, data and evidence have been cumulating, in support of the feasibility and validity of plasma pharmacological method in medicinal herb ex vivo research.

Plasma Samples Reflect the Levels of Absorbed Herbal Components Some researchers had successfully used plasma in medicinal herb studies such as measurements of effective components, ${ }^{16)}$ protein binding rate ${ }^{17)}$ and pharmacokinetics. ${ }^{18)}$ Recent study showed in a rabbit model that after administration of Danggui (a traditional Chinese medicine, TCM), more than 32 common components were detected in both the Danggui oral solution used for administration and the plasma sample, with at least 10 new components detected solely in the plasma sample. ${ }^{19)}$ These findings strongly indicate that plasma samples maintain the integrity of the original herbal components, their metabolites and/or endogenous substances induced by medicinal herb administration.

Levels of Absorbed Herbal Components between Plasma and Serum May Be Different Based on the published literature of serum and plasma pharmacology, drugs ( single component) can be divided into three categories: (1) plasma level higher than serum level, such as clozapine, ${ }^{20)}$ imipramin ${ }^{21)}$; (2) plasma level lower than serum level, e.g. aminoglycoside, ${ }^{22)}$ mefloquine ${ }^{23)}$; (3) plasma level and serum level close, such as voriconazine, ${ }^{24)}$ topiramate. ${ }^{25)}$ It is inferable that the differences and similarities of herbal components between serum and plasma are analogous to the single component drugs mentioned above. Unfortunately, there are limited reports available in the current literature on serum and plasma herbal components comparison. Our study using HPLC-MS combined with fingerprint profile analysis after feeding Tailaofang decoction (a TCM) in rats showed some marked differences between the plasma and serum samples (Fig. 2). Particularly, most components in the polar portion of the serum HPLC-Photo Diode Array (PDA) chromatogram were markedly lower than those in the plasma chromatogram $^{8)}$ (Fig. 3). Other laboratory also reported similar findings that after feeding Wurenchun decoction (a TCM) to rats, the level of effective herbal components (schisandrin A and B) is significantly higher in plasma than in serum samples. $^{26)}$

Respondents of Herbs in Plasma and Serum Samples Might Not Completely Match By proteomic analysis, Stroncek et al., followed changes in 80 proteins after granulocyte colony-stimulating factor (G-CSF) stimulation in healthy adults. They showed that prior to G-CSF treatment, 44 proteins levels were different between serum and plasma. Following G-CSF treatment, changes are seen in 14 proteins in serum and 15 proteins in plasma respectively. Among them, 11 proteins were upregulated in both serum and plasma, and the changes of 4 proteins were plasma-specific and 3 proteins were serum-specific. ${ }^{27)}$

However, as discussed earlier, the liquid portion of circulating blood in vivo is plasma rather than serum. If serum is exclusively being used in pharmacological studies, the conclusions drawn are highly likely to be flawed. Following a parallel paired design, we recently used a rat model to study serum and plasma responses to a modified Buyanghuangwu decoction (a TCM). 664 total protein spots were analyzed by two-dimensional gel electrophoresis, and 76 and 69 spots were serum-specific and plasma-specific repectively. ${ }^{28)}$ These data suggest that after medicinal herb administration the respondent components in serum and plasma shares similarity as well as disparity.

Bioactivity of Herbs May Be Different between Plasma and Serum Samples Huang et al., ${ }^{29)}$ explored the effects of Jiexinkang (a TCM) on leukemic cells using serum samples containing absorbed Jiexinkang. When the serum absorbed-Jiexinkang was added at increasing concentrations from 2.5 to $10 \%(\mathrm{v} / \mathrm{v})$ in their ex vivo experiment, dosedependent inhibitory effects were observed in leukemic cell proliferation. Interestingly, higher concentrations from 10 to $20 \%(\mathrm{v} / \mathrm{v})$ exhibited opposite effect and promoted leukemic cell proliferation dose-dependently. In the serum control group, serum alone promoted leukemic cell proliferation in a 

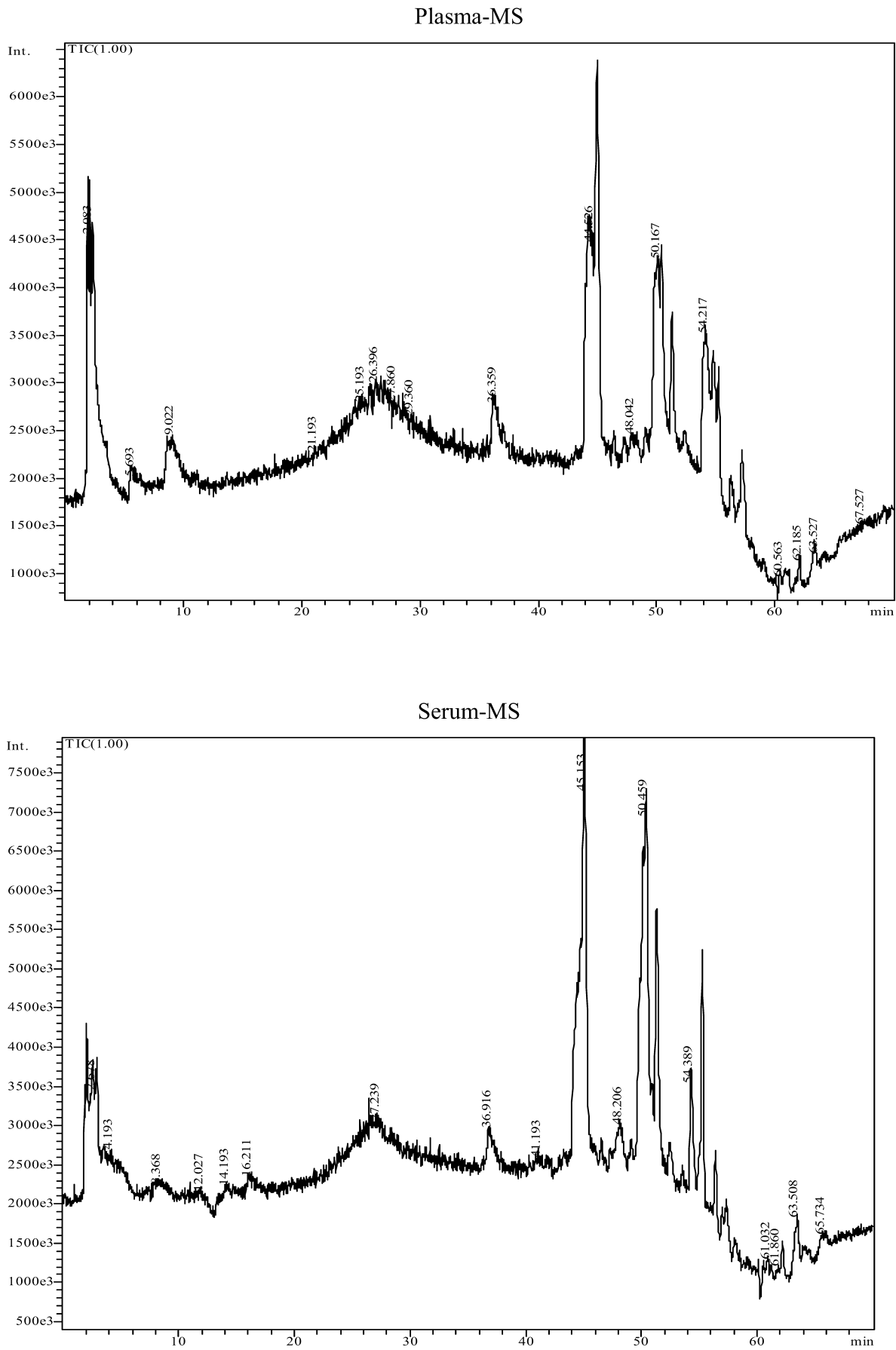

Fig. 2. Comparison of Mass Spectrometry Chromatogram between Plasma (Upper) Absorbed Tailaofang (a Traditional Chinese Medicinal Compound) and Serum (Lower) Absorbed Tailaofang

dose-dependent manner in the dose range of 2.5 to $20 \%$ $(\mathrm{v} / \mathrm{v})$. The promotive effect seen in serum is mainly attributable to the richness of growth factors and cytokines released after coagulation and subsequent platelet and leukocyte activation. It is obvious that the inhibitory effect of Jiexinkang on leukemia cell proliferation was mitigated by the stimulatory effect of the serum base, accentuating the flaws of the serum pharmacological method.

Promising data of plasma pharmacology has been emerging lately. In platelet aggregation assays, we observed markedly higher inhibitory effect of absorbed Dahuangzhechong (a TCM) in the plasma samples in our parallel runs of both plasma and serum samples. ${ }^{30)}$ He and Zhou, recently reported more potent proangiogenic effect of absorbed Danshan (a TCM) in plasma samples compared to serum samples in chorioallantoic membrane vascular assays. ${ }^{31)}$ Luo et al., showed markedly higher protective effect of plasma absorbed Wurenchun (a TCM) on injured hepatocytes than serum absorbed Wurenchun. ${ }^{32)}$ In addition, we used plasma pharmacological method to test the effect of Tongxinluo (a TCM) on vascular endothelial cells, and observed remarkable inhibitory effect on the ex vivo expression of tissue factor and angiotensin II receptor subtype $1 .^{33)}$ Recently Li et al., also successfully demonstrated that total Panax Notoginseng 
Plasma-PDA

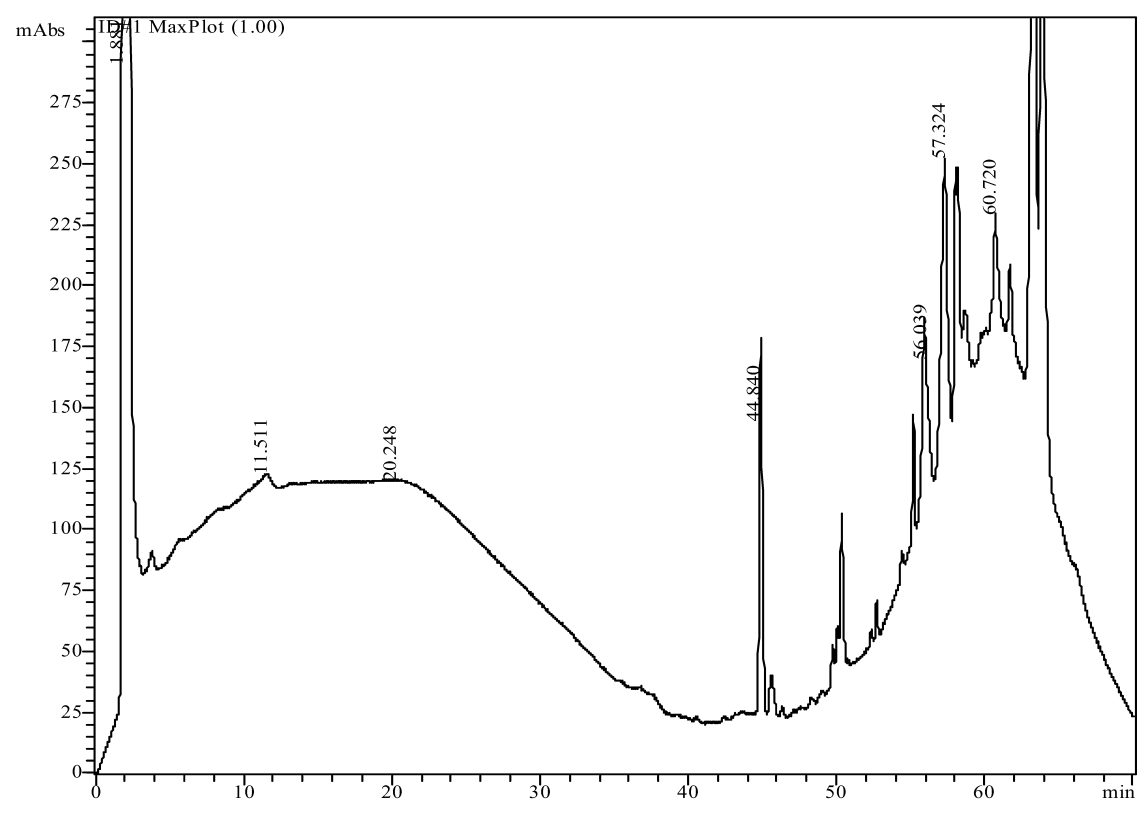

Serum-PDA

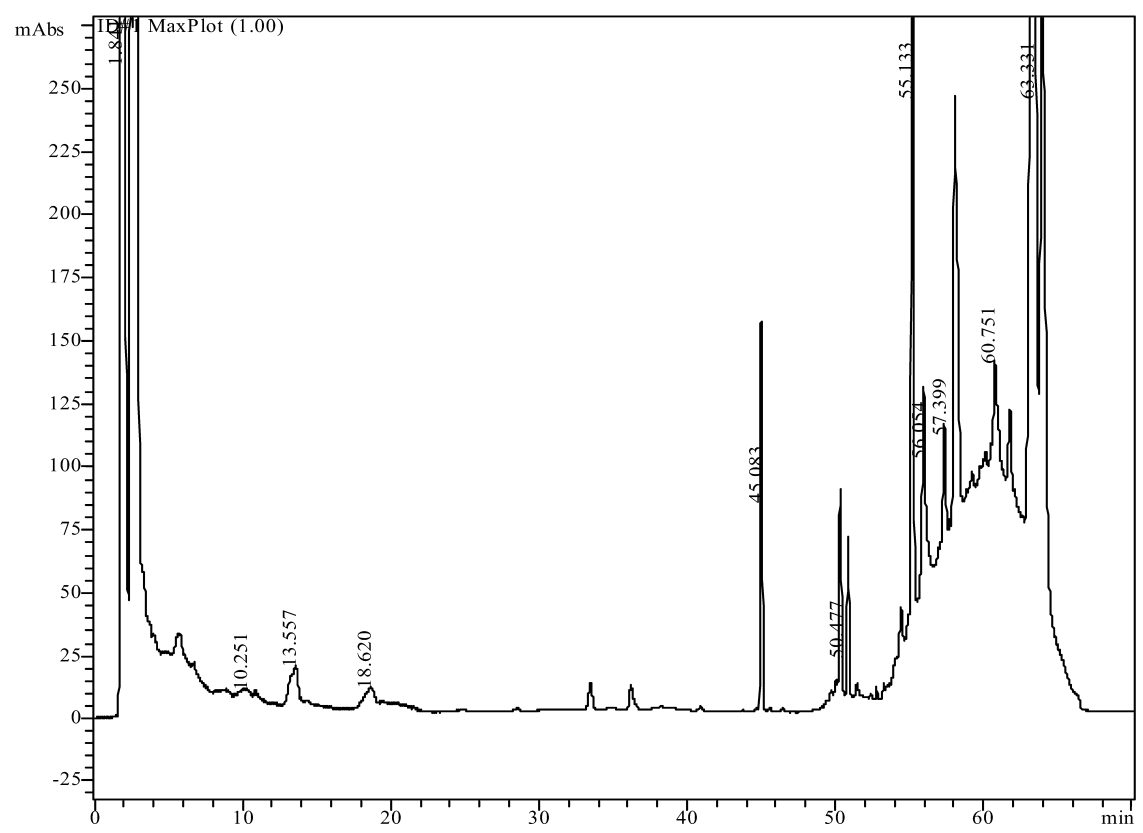

Fig. 3. Comparison of PDA Chromatogram between Plasma (Upper) Absorbed Tailaofang (a Traditional Chinese Medicinal Compound) and Serum (Lower) Absorbed Tailaofang

saponins (a TCM) markedly inhibited PDGF-induced vascular smooth muscle cell proliferation in vitro using plasma pharmacological method. ${ }^{34)}$ These promising reports strongly support the feasibility and validity of plasma pharmacological methods.

\section{CONCLUSIONS AND FUTURE PERSPECTIVES}

Confronted with the uncertainty of conclusions drawn from serum pharmacology method due to its systemic flaws, and the early developmental stage of plasma pharmacological method, more and more researchers began to wonder which method is the better choice for ex vivo herb research. This is an important and fundamental question for the science of medicinal herb pharmacology. Tremendous amount of work are needed to satisfactorily and convincingly answer this question. At the current stage, we would like to propose the following suggestions:

Advocate Plasma Pharmacological Method in ex Vivo Experiments It is imperative that the constituents of body fluid being tested remain as unchanged as possible from their in-vivo states to obtain valid medical experiment results. As recent WHO document ${ }^{35)}$ pointed out: "Historically, serum was the preferred assay material for determining extracellular 
concentrations of constituents in blood. Today, plasma is preferred for many, but not all, laboratory investigations because the constituents in plasma are better reflecting the pathological situation of a patient than in serum." Advantages of using plasma versus serum mainly include: (1) The constituents of plasma are much closer to the in vivo state of the cell-free portion of circulating blood, due to the absence of interferences from coagulation and its related processes (activation of fibrinolysis, kinin and complement systems, release of platelets and leukocytes as well as injury of erythrocytes) as seen in serum. (2) The volume yield of plasma is 15 to $20 \%$ higher than that of serum from the same amount of blood. (3) The preparation of plasma is time-saving requiring only centrifugation. Thus, even though plasma pharmacology method is currently not as widely used or fully developed as the serum pharmacology method, they should not be ignored any more, and their significant role in medicinal herb studies should be pursued and explored.

Launch Studies to Identify Applicable Fields and Appropriate Anticoagulants for Plasma Pharmacological Method The anticoagulants currently used in vitro are chelate, heparin and hirudin. Chelating agents including ethylenediamine tetraacetic acid (EDTA), oxalate, and citrate work through binding and removal of calcium ion, which is indispensable for multiple enzyme reactions in the coagulation cascade. The chelating capability is EDTA $>$ oxalate $>$ citrate. Heparin inhibits several serine proteases (thrombin, FXa, FIXa, etc.) via binding antithrombin but it is procoagulant in the absence of antithrombin. ${ }^{36)}$ Hirudin is a direct and specific thrombin inhibitor. Generally speaking, EDTA is widely used in studies of proteins, peptides, cytokines, other labile molecules and molecular biology. ${ }^{37)}$ Heparin is usually used in ex vivo experiments that are calcium-dependent. Citrate is commonly used in hematology, especially coagulation studies. Hirudin can be used for complement measurement and certain cellular expression assays. ${ }^{38)}$ It is worth mentioning that proteomic studies have demonstrated differences not only between serum and plasma, but also between plasma samples prepared with different anticoagulants. ${ }^{39)}$ Thus, if plasma pharmacological method is used in an ex vivo experiment, what is the most appropriate anticoagulant for it? It is highly possible that no single anticoagulant can be used universally in all experiments, and different constituents/ingredients of herbs and different functional tests would call for different anticoagulants.

Adopt Appropriate Attitude towards Serum Pharmacological Method The fact that we point out the flaws of the serum pharmacological method does not mean we totally object to serum pharmacological method. Actually both serum and plasma pharmacological methods have their merits depending on the usages, i.e. both methods are complementary. It is necessary to add anticoagulant into blood for preparation of plasma. However, anticoagulant may interfere with certain experiments. For example, EDTA inhibits $\mathrm{Ca}^{2+}$ dependent reaction, heparin inhibits Taq polymerase in PCR, and fibrinogen interferes with immunoassays. Therefore, there is full scope for usage of serum pharmacological method when anticoagulants can not be used. If the effects of coagulation and/or anticoagulation on medicinal herbs pharmacological testing are not fully understood, it may be prudent to run serum and plasma samples parallelly. Consistent overall trends observed from both methods would provide powerful support and reassurance for the validity of the data and conclusion. In case the data are contradictory to each other, the next step should be repeating the experiment with plasma samples prepared with different anticoagulants, as plasma samples are better reflectors of vivo state. If interference from an anticoagulant is suspected, the anticoagulant can be added into the corresponding serum samples to further evaluate the suspected effect of the anticoagulant, helping to reach a correct conclusion.

In summary, the constituents of plasma better reflect the physiological and pathological status in vivo than the constituents of serum. Plasma pharmacological method should be recommended and further explored for ex vivo experiments in medical herb research. Studies need to be launched to identify applicable fields and appropriate anticoagulants for plasma pharmacological methods. However, serum pharmacological method should be used when anticoagulants interfere with experiment. In certain conditions, plasma and serum pharmacological methods might need to be used parallelly to obtain the correct conclusion and unveil the disguised truth.

Acknowledgements This work was supported by Natural Sciences Foundation of China (Grant No. 30572370, J. Ge; and No. 30572455, 30670990, D. Wang). We thank Professor Xiu Chen for reviewing this manuscript.

\section{REFERENCES}

1) Iwama H., Amagaya S., Ogihara Y., J. Ethnopharmacol., 21, 45-53 (1987).

2) Chen N. N., Huang S. L., Xiang Y., Zhang D. J., Guo A. X., Chen A. P., J. Chin. Integr. Med., 5, 65-69 (2007).

3) Yaqmamoto K., Zhang J. P., Yamamoto M., Yamamoto N., Kanai M., Aoyagi M., Zhang B. L., Biol. Pharm. Bull., 27, 1010-1013 (2004).

4) Amagaya S., Harada K., Miyake A., Iwama H., Ogihara Y., Chem. Pharm. Bull., 37, 1117-1119 (1989).

5) Zhao B. S., Huo H. R., Liu H. B., Ma Y. Y., Li L. F., Li C. H., Guo S. Y., Guo J. Y., Jiang T. L., J. U.S.-China Med. Sci., 4, $61-67$ (2007).

6) Liu F., Wang J. G., Wang S. Y., Li Y., Wu Y. P., Xi S. M., World J. Gastroenterol., 14, 3990-3996 (2008).

7) He R., "Medical Research Methodology," 1st ed., ed. by He S. L., Li Y. J., People's Military Medical Press, Beijing, 2003, pp. 411- 420.

8) He S. L., Ge J. W., He R., Mei Z. G., Chin. Pharmacol. Bull., 21 $277-279$ (2005).

9) Ge J. W., Zhu H. B., Wang Y. H., He R., He S. L., World Sci. Technol./Modern TCM Meteria Med., 10, 16-22 (2008).

10) Brass L. F., Furie B., Lijnen H. R., Esmon C. T., "Hematology: Basic Principles and Practice," 3rd ed., ed. by Hoffman R., Benz E. J., Shattil S. J., Furie B., Cohen H. J., Silberstein L. E., Churchill Livingstone, New York, 2001, pp. 1753-1824

11) He S. L., Ge J. W., J. Diagnost.: Conc. \& Prac., 4, 433-434, 513 516 (2005).

12) Amara U., Rittirsch D., Flierl M., Bruckner U., Klos A., Gebhard F., Lambris J., Huber-Lang M., Adv. Exp. Med. Biol., 632, 71-82 (2008).

13) Thadikkaran L., Siegenthaler M. A., Crettaz D., Queloz P. A., Schneider P., Tissot J. D., Proteomics, 5, 3019-3034 (2005).

14) Tammen H., Schulte I., Hess R., Menzel C., Kellmann M., Mohring T., Schulz-Knappe P., Proteomics, 5, 3414-3422 (2005).

15) Ayache S., Panelli M. C., Byrne K. M., Slezak S., Leitman S. F., Marincola F. M., Stroncek D. F., J. Trans. Med., 4, 40-51 (2006)

16) Wang X. J., Sun H., Fan Y. L., Li L. J., Makino T., Kano Y., Biol. Pharm. Bull., 28, 1106-1108 (2005)

17) Huang H., Liu H., Liang Q., Lin M., J. Guangxi. Med. Univ., 25 $169-173$ (2008)

18) Gao Q. T., Chen X. H., Bi K. S., Biol. Pharm. Bull., 27, 226-228 
(2004).

19) Wang Y., Liang Y., Chen B., He Y., Li B., Hu Q., Anal. Bioanal. Chem., 383, 247-254 (2005).

20) Kaladjian A., Bery B., Deturmeny E., Bruguerolle B., Ther. Drug Monit., 21, 327-329 (1999).

21) Hullett F. J., Levy A. B., Tachiki K. H., J. Clin. Psych., 45, 516-518 (1984).

22) Ebert S. C., Leroy M., Daxcey B., Ther. Drug Monit., 11, 44- 46 (1989).

23) Todd G. D., Buma A. P., Green M. D., Jaspers C. A., Lobel H. O., Am. J. Trop. Med. Hyg., 57, 399- 402 (1997).

24) Langman L. J., Boakye-Agyeman F., Clin. Biochem., 40, 1378-1385 (2007).

25) Berry D. J., Patsalos P. N., Ther. Drug Monit., 22, 460 - 464 (2000).

26) Luo L., Dou Z. H., Ding A. W., Wang L. J., Zhang B., Shi Z., Chin. Trad. Herbal Drugs, 37, 1486-1489 (2006).

27) Stroncek D., Stezak S., Khuu H., Basil C., Tisdale J., Leitman S. F, Marincola F. M., Pennelli M. C., Exp. Hematol., 33, 1109-1117 (2005).

28) Ge J. W., Zhu H. B., Wang Y. H., Chen Y., Wang G., He S. L. (unpublished).

29) Huang C. H., Xie Z. X., Qin Q., Lin X. M., Bull. Hunan Medical
Univ., 28, 477-480 (2003).

30) Wang D. S., Chen F. P., He S. L., Xie A. Z., Fu B., Li X., Cao X. Y., Chen Y., Chin. J. Thromb. Haemost., 11, 5-8 (2005).

31) He L., Zhou L., Trad. Chin. Drugs Clin. Pharmacol., 18, 431- 433 (2007).

32) Luo L., Dou Z., Wang F., Xu Z., Pharmacol. Clin. Chin. Materia Med., 24, 53-55 (2008).

33) Ma Q. L., Sun M., Yang T. L., Li Y. J., Tang C. E., Peng Z. Y., He S. L., Chen F. P., J. Cent. South Univ. (Med. Sci.), 32, 485-489 (2007).

34) Li H., Chen Q., Deng C. Q., J. Chin. Integr. Med., 7, 1078-1085 (2009).

35) WHO, "Use of Anticoagulants in Diagnostic Investigation," WHO/DIL/LAB/99.1 Rev. 2 (2002).

36) Banfi G., Salvagno G., Lippi G., Clin. Chem. Lab. Med., 45, 565-576 (2007).

37) Smith S. A., Morrissey J. H., Thromb. Haemost., 100, 160-162 (2008).

38) Bexborn F., Engberg A., Sandholm K., Mollnes T., Hong J., Ekdahl K. N., J. Biomed. Materia Res., 89A, 951—959 (2009).

39) Kim H. J., Kim M. R., So E. J., Kim K. W., J. Biochem. Biophys. Methods, 70, 619-625 (2007). 